\title{
A Quantitative Methodology for the Study of Particle- Electrode Impacts
}

\begin{tabular}{|c|c|}
\hline Journal: & Physical Chemistry Chemical Physics \\
\hline Manuscript ID & CP-ART-03-2018-001561.R1 \\
\hline Article Type: & Paper \\
\hline Date Submitted by the Author: & 18-Apr-2018 \\
\hline Complete List of Authors: & $\begin{array}{l}\text { Little, Christopher; Oxford University, Department of Chemistry } \\
\text { Xie, Ruochen; Oxford University, Department of Chemistry } \\
\text { Batchelor-McAUley, Christopher; Oxford University, Department of } \\
\text { Chemistry } \\
\text { Kätelhön, Enno; Oxford University, Department of Chemistry } \\
\text { Li, Xiuting; University of Oxford } \\
\text { Young, Neil; Oxford University, Department of Materials } \\
\text { Compton, Richard; Oxford University, Department of Chemistry }\end{array}$ \\
\hline
\end{tabular}




\title{
A Quantitative Methodology for the Study of Particle-Electrode Impacts
}

Christopher A. Little ${ }^{\star}$ Ruochen Xie,${ }^{\star}$ Christopher Batchelor-McAuley, Enno Kätelhön, Xiuting Li, Neil P. Young and Richard G. Compton*

* corresponding author: Richard G. Compton, Department of Chemistry, Physical \& Theoretical Chemistry Laboratory, Oxford University, South Parks Road, Oxford, OX1 3QZ, United Kingdom

${ }^{\ddagger}$ C.A.L. and R.X. contributed equally

Email: richard.compton@chem.ox.ac.uk. Tel: +44(0)1865275957 Fax: +44(0)1865275410

\begin{abstract}
Herein we provide a generic framework for use in the acquisition and analysis of the electrochemical responses of individual nanoparticles, summarising aspects that must be considered to avoid mis-interpretation of data. Specifically, we threefold highlight the importance of the nanoparticle shape, the effect of the nanoparticle diffusion coefficient on the probability of it being observed and the influence of the used measurement bandwidth. Using the oxidation of silver nanoparticles as a model system, it is evidenced that when all of the above have been accounted for, the experimental data is consistent with being associated with the complete oxidation of the nanoparticles (50 $\mathrm{nm}$ diameter). The duration of many single nanoparticle events are found to be ca. milliseconds in duration over a range of experiments. Consequently, the insight that the use of lower frequency filtered data yields a more accurate description of the charge passed during a nanoevent is likely widely applicable to this class of experiment; thus we report a generic methodology. Conversely, information regarding the dynamics of the nano redox event is obscured when using such lower frequency measurements; hence, both data sets are complementary and are required to provide full insight into the behaviour of the reactions at the nanoscale.
\end{abstract}




\section{Introduction}

The technique of particle coulometry has continued to evolve in the last 50 years; ${ }^{1-2}$ the last decade in particular has seen rapid development in this field with research focusing on the characterisation of individual nanoparticles. ${ }^{3-4}$ The technique has been applied to the study of a variety of nanomaterials including gold, silver, copper, $\mathrm{Fe}_{3} \mathrm{O}_{4}$ and liposomes. In general, an electrode is immersed into a solution containing a redox active nanoparticle suspension. The nanoparticles move around in solution by virtue of their Brownian motion. Upon making electrical contact with or 'impacting' a suitably potentiostated electrode, the particle may be oxidised or reduced, resulting in its destruction. These nanoparticle-electrode impacts are herein referred to as 'nano-impacts'. The oxidation or reduction of a single nanoparticle results in a single oxidative or reductive transient feature in the current-time trace. In this work, such current transients will be referred to as 'spikes'. Due to the often small size (ca. picoamperes) of current involved in these reactions, consideration of the signal-to-noise ratio is important and can limit the size of the smallest observable features. ${ }^{5}$ Measurement of the current passed during this redox event yields a direct measure of the number of electrons transferred during the process. For a redox reaction of known stoichiometry, with the use of Faraday's first law and often under the assumption that the density of the material is the same as that of the bulk, a direct estimate for the volume of material oxidised during the event can be obtained. The measurement of the charge passed during the event facilitates the estimation of the volume of material oxidised or reduced; this electrochemically determined volume can be compared to other particle sizing techniques in order to establish the extent of oxidation (or reduction) of the particle that has occurred during the course of a 'single' impact event.

Electron microscopy is widely accepted as the optimal method for particle sizing. The specific technique required depends on the size of the particles being studied. Whilst Scanning Electron Microscopy (SEM) is appropriate for larger particles, for diameters smaller than tens of nanometres the use of Transmission Electron Microscopy (TEM) is necessitated to ensure a higher level of accuracy. Moreover, as will be highlighted below, the requirement of obtaining and fully accounting for shape information regarding a nanoparticle sample subsequently means that only the family of TEM based techniques have sufficient resolution to enable comparison to be made with experimental electrochemical data. Even with the use of TEM, major ambiguities arise in the estimation of a nanoparticle volume distribution. Techniques are available for the use of TEM to improve the $3 \mathrm{D}$ estimation of the particle shape either through tomography ${ }^{6}$ or by "counting, ${ }^{7}$ the number of atoms present in individual particles. This affords an improved estimate of the particle volume distribution. Whilst this atom counting is well suited to very small particles, larger particles pose a greater challenge. 
A number of subtleties in comparing conventional 2D microscopy and electrochemical data need to be highlighted. First, microscopy techniques are not devoid of error; the sizing information obtained is sensitive to both the magnification at which the images have been obtained, and the operator. ${ }^{8}$ An intrinsic artefact of TEM imaging in this context is the presence of defocusing rings surrounding a particle. The ambiguity from the blurring of the edges of the particles is compounded by variations in contrast of the sample with the support, and the subsequent thresholding employed when analysing the images. The resultant error in radius measurement leads to an error in the estimated volume of a particle. Moreover, this error in the estimated volume is proportional to the cube of the error in the measured radius. Estimation of a particle volume from a 2D image necessarily requires assumptions to be made regarding the particle geometry. Challengingly for microscopy, the circularity of a 2D projection of a particle is not a good estimate of its sphericity. Consequently, even for a solid with a high degree of symmetry and circularity in its 2D projection such as an icosahedron, its volume can differ very markedly from that of the circumscribed sphere $(60.5 \%$ in the case of an icosahedron). ${ }^{9}$ The equilibrium particle shapes of crystalline solids are highly faceted and can be described using a Wulff construction. ${ }^{10}$ However, the actual morphology of a particle sample depends on a wide variety of factors including synthesis method, aging and particle size. Even before the weak confinement limit (ca. $<2 \mathrm{~nm}$ for metallic materials ${ }^{11}$ ) is reached, the properties of the solid may differ from that of the bulk material due to the increased surface energy. ${ }^{12}$ Such surface curvature effects become influential for silver and gold particles at diameters approximately $<20 \mathrm{~nm} \cdot{ }^{13-14}$ As a result, nanoparticle samples often exhibit a wide variety of shapes and sizes. A given population of particles cast onto a TEM grid will consequently exhibit a diverse array of structural motifs, complicating statistical analysis of sizing. Ultimately, the pragmatic approximation of a particle as a sphere is a poor estimate of a particles true $3 \mathrm{D}$ volume; this stems from the variation of polyhedral shapes and the presence of faceting of the particles.

Second, the electrochemical data reports on the events that have occurred at an electrochemical interface. The probability of a particle arriving at a surface is a function of its diffusion coefficient; for truly monodispersed samples this only serves to alter the expected impact frequency. However, for polydispersed nanoparticle populations (all nanoparticle populations exhibit some degree of heterogeneity) this leads to the electrochemical method being biased towards the observation of smaller particles. This arises due to the smaller particles moving faster within the solution and they are therefore more likely to impact the electrochemical interface. The issue is compounded to an extent by the propensity for nanoparticles to agglomerate in the solution phase in the presence of an electrolyte. Hence, such experiments are now regularly performed using only millimolar salt concentrations. ${ }^{15}$ A further related issue is the tendency for particles to adsorb on to surfaces (such 
as glass) surrounding the electrode. This is especially important when considering sample contamination. Often, significant efforts need to be undertaken to avoid cross contamination of nanoparticle samples.

Third, measurement and subsequent analysis of the electrochemical data can significantly influence the resulting volume distribution. Recent work in the literature has highlighted the benefits of recording the electrochemical impact data with relatively high bandwidths (ca. $10 \mathrm{kHz})^{16-18}$. However - as a note of caution - it is highlighted that work utilising scanning electrochemical cell microscopy (SECCM) is potentially undermined by plausible nanoparticle contamination from the used quasi-reference electrode. ${ }^{19-20}$ This caveat aside, at higher frequencies individual nanoparticleelectrode impact events are revealed to be comprised of multiple features. However, the shape of many of these events and the magnitude of the measurement noise are influenced by the used measurement and filtering system. ${ }^{21}$ Any measurement system necessarily provides a non-perfect representation of the process it is recording. For electrochemical systems, one of the most important parts of the device is the current-amplifier. A transimpedance amplifier is often employed in the measurement of small currents, but the bandwidth of this device is often limited due to the high gains used. ${ }^{22}$ The current-amplifier simultaneously acts in two ways. First, it behaves as an amplifier, converting the current into a voltage output at a predetermined gain, commonly $\sim 1 \mathrm{GV} / \mathrm{A}$. Second, it acts as a filter, the transfer function of which is often designed to be first order and lowpass. In the study of nanoparticle-electrode impact experiments, many nano-reactions occur at rates faster than can be accurately reported by the amplifier. Consequently, the shape of the resulting measured nano-impact signal is often found to be influenced by the bandwidth and transfer function of the measurement system. In some cases, the nano-impact event may be so fast (relative to the bandwidth of the used electronics) that the resulting spike shape is that of the measurement systems impulse response. ${ }^{21}$ However, although the spike shape can reflect the transfer function of the measurement system, the integral under the current trace, the value of which is equal to the charge passed during the impact, is conserved, assuming the system has been suitably designed.

Beyond considering how the representation of a nano-impact event changes with the used measurement frequency, it is important to consider how a systems electrical noise and hence the measured signal-to-noise ratio changes as a function of the signal bandwidth. As a simplified answer to this problem, assuming the background noise is similar to that found for an idealised resistor (Johnson-Nyquist, i.e. the power spectral density is independent of the frequency) then the standard deviation of the noise current will vary linearly with the square-root of the signal bandwidth. ${ }^{23}$ In contrast, the impulse response of a system is linearly proportional to the signal bandwidth. Consequently, one may anticipate the systems signal-to-noise ratio to improve as the measurement systems frequency (bandwidth) is increased. However, this will only hold true if first, 
the power spectrum is flat - for an electrochemical system the noise power spectral density will tend to be greater at higher frequencies due to the capacitative coupling of the electrode to the systems voltage noise. ${ }^{5}$ Second, if the current measured for a nano-impact event is not that of the impulse response of the system, such that the kinetics of the nano-impact processes are at least partially resolved by the measurement system, then the spike height will not increase linearly as a function of the measurement frequency. Consequently, as will be demonstrated in this article, in the most extreme case the use of high bandwidth measurement can result in the complete loss of information as the processes of interest are obscured by the noise of the system. Further considerations detailing the importance of signal-to-noise ratios in electrochemical measurements have been highlighted elsewhere. $^{24-25}$

Aside from the issues of the measurement of the signal-to-noise ratio, the use of higher measurement frequencies also raises the questions of what constitutes a 'single' electrochemical event and how might the bandwidth at which the data is analysed influence the resulting size distribution? To further clarify terminology, as mentioned above, in this work the term 'spike' refers to a single transient oxidative feature observed in the current-time trace at a given data filtering frequency. During the course of the spike, the measured current does not pass through a pre-defined baseline, but a single spike may exhibit multiple maxima and minima. Likewise, a single nano-impact event is taken to be one or more spikes in current that occur close to each other in time. The clustering of individual spikes into a single 'nano-event' may be justified on the basis of the known or expected frequency of the nano-impact events, such that the probability that two or more spikes are independent of each other can be defined statistically. The purpose of this article is to systematically explore the question of how the bandwidth at which the experimental data is presented and analysed influences both the qualitative and quantitative conclusions made for a nano-impact experiment. To answer this question, the oxidation of silver nanoparticles (AgNPs) is taken as a model nanoparticle coulometry system. However, the results have significant wider implications for a range of electrochemical single entity studies.

\section{Results and Discussion}

A carbon microdisc electrode (radius $=16.5 \mathrm{um}$ ) was immersed into a solution containing a $12 \mathrm{pM}$ suspension of silver nanoparticles (diameter $=50 \mathrm{~nm}$ ) and $20 \mathrm{mM} \mathrm{KCl}$. SI section 1 provides TEM images of the silver nanoparticles. Application of an oxidising potential to the electrode $(+0.8 \mathrm{~V}$ vs SCE) and measurement of the current resulted in the observation of spikes in the time-current trace. Figure 1 depicts a representative chronoamperogram, as measured over twenty seconds. In the absence of solution phase nanoparticles these fluctuations or spikes in the current were not observed. 
As has been previously documented, ${ }^{26}$ these spikes in current are associated with the arrival and electrochemical oxidation of individual nanoparticles at the electrochemical interface. In this experiment, the bandwidth of the analog signal was $2 \mathrm{kHz}$. The current amplifier had a bandwidth of $4 \mathrm{kHz}$ and the output was subsequently low-pass filtered (analog) prior to digitalisation using two cascaded passive RC filters to limit the analog signals frequency to $2 \mathrm{kHz}$. The choice to filter a signal before it is digitised often arises due to limitations in the available sampling rate. As a minimum, the digitalisation sampling rate needs to be twice the systems measurement frequency (Nyquist-Shannon $^{27-29}$ sampling theorem); however, in the present case we are interested in the charge passed during a single nano-impact event. Consequently a far higher oversampling rate is required to ensure the analog signal is accurately represented in the digital data. In the present work, the $2 \mathrm{kHz}$ analog signal is sampled at $100 \mathrm{kS} \mathrm{s}^{-1}$, a factor of 50 times faster. It is important that the bandwidth (frequency) of a signal and the rate at which it is digitised (sampling rate) are not conflated. A flow diagram detailing the used experimental measurement system is depicted in the SI section 2. Following digitisation, the signal is stored and can be digitally filtered (4-pole Bessel) as required following its acquisition. The use of a digital filter readily allows the same experimental data set to be viewed at different signal bandwidths and allows, in this work, for the influence of the filtering to be readily explored and exemplified.

As shown in Figure 1 (black line) the oxidative spikes in current are superimposed upon a relatively slowly changing background current. During the chronoamperogram, the background current value changes from $\sim 10 \mathrm{nA}$ at short times to less than $1 \mathrm{nA}$ towards the end of the 20 second scan. This background current is ascribed to the capacitative charging of the electrode and at higher electrode potentials also includes a noticeable Faradaic contribution arising from solvent breakdown. Analysis of individual spikes requires their delineation from the slowly-changing background current. A number of techniques and methods may be developed for this purpose; in the present work we utilise a method in which the local (windowed) variance of the current response is calculated andareas of high local variance are removed from the chronoamperogram prior to estimation of the baseline. Baseline estimation is then achieved though low-pass filtering of the time-current transient. Further information on the employed methodology is provided in the SI section 3. Having obtained an estimate of the slowly varying background signal, this baseline transient is subtracted from the data, to give a baseline-subtracted time-current trace, as shown in Figure 1 (red line). Notably the quantification of the integral of a spike is sensitive to the accuracy and quality of the baseline estimation and is an important factor in the analysis of the experimental data. 




Figure 1. Representative chronoamperogram of $12 \mathrm{pM} \mathrm{AgNPs} \mathrm{in} 20 \mathrm{mM} \mathrm{KCl}$ at a $16.5 \mu \mathrm{m}$ radius carbon microdisc electrode in which the potential is held at $0.8 \mathrm{~V}$ (vs. SCE) for $20 \mathrm{~s}$. The black line depicts the raw data, with the baseline subtracted data overlaid in red.

After removal of the low frequency baseline, the individual spikes are still superimposed upon a variable higher frequency (noisy) background. The specific origin of these high frequency background fluctuations in current depends upon the used experimental set-up; the electrical noise is commonly dominated by thermal fluctuations at the electrochemical interface or in the used current amplifier. ${ }^{23}$ Having removed the slowly changing background, spike identification becomes a simpler task. In this work, a method is utilised in which a spike is identified and begins when the measured current passes through a threshold value, finishing when the current returns to the baseline. The magnitude of the used threshold can be set by the user, but is most commonly taken as either three or five times the estimate of the standard deviation of the background noise current. The start and end of the spike are subsequently defined by the points at which the current passes through the calculated baseline.

Figure 2 depicts a section of experimental raw data with the slowly-changing baseline removed, overlaid is the same data where it has been subsequently digitally low-pass filtered post-acquisition to $500,100,50$ and $25 \mathrm{~Hz}$ using a four-pole Bessel filter. Decreasing the signal bandwidth leads to a reduction in both the spike height and background noise. The width (duration) of the spike in current also increases with decreasing frequency, this is true to the extent that two individual spikes as seen in the raw data with maxima at 11.510 and 15.267 seconds are merged into a single event at $25 \mathrm{~Hz}$. 




Figure 2. Representative baseline-subtracted chronoamperogram impact spikes for $12 \mathrm{pM}$ AgNPs in $20 \mathrm{mM} \mathrm{KCl}$ at a carbon microdisc electrode ( $33 \mu \mathrm{m}$ diameter). The black line shows the raw data and the red, yellow, blue and green lines show the effect of filtering at 500, 100, 50 and $25 \mathrm{~Hz}$, respectively.

This result highlights how filtering the data can lead to a loss of information as individual spike events can become merged into single events when filtered at a low frequency. Conversely, the use of higher filter frequencies can also lead to obfuscation of features in the data. Figure 3 depicts more data both in its raw (not digitally filtered) state and the same data when filtered digitally to $100 \mathrm{~Hz}$. In the raw data shown in Figure 3 a), only one spike event can be identified on the basis of the current increasing significantly above the background noise; however, the same data when filtered to $100 \mathrm{~Hz}$ shows a secondary peak approximately $30 \mathrm{~ms}$ after the first. This experimentally highlights how slow electrochemical processes can be lost (nominally 'vanish') into the data's noise when viewed at higher frequencies. Consequently, the experimental data can appear qualitatively different depending on the frequency at which it is viewed. Ultimately, as the measurement frequency is increased, at sufficiently high bandwidths all Faradaic data will become lost in the systems noise. 

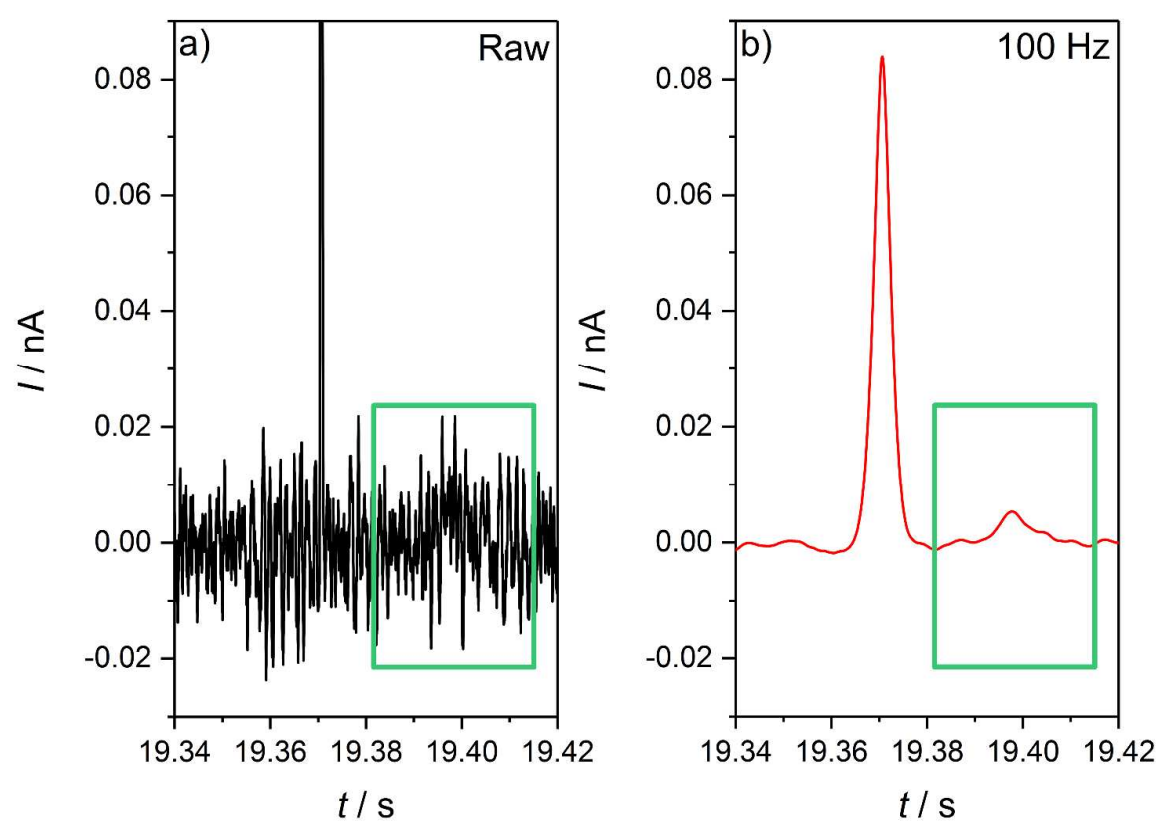

Figure 3. Representative baseline-subtracted example of a 'vanishing' spike for $12 \mathrm{pM} \mathrm{AgNPs} \mathrm{in} 20 \mathrm{mM} \mathrm{KCl}$ at a carbon microdisc electrode ( $33 \mu \mathrm{m}$ diameter). The raw data is shown on the left with the region of the 'vanishing' spike outlined in green, and the same data filtered at $100 \mathrm{~Hz}$ is shown on the right. The charge of this 'vanishing' spike at 100 $\mathrm{Hz}$ is $0.038 \mathrm{pC}$.

For many applications in particle coulometry, measurement of the charge passed during a single nano-impact event is of interest. If we take the duration of a single event to be defined as when the current leaves and returns to a pre-determined baseline, we can consider how the measured charge distribution alters as the data is analysed at different frequencies. Hence, the charge per spike event was measured for the same data set but where the data has been digitally filtered prior to spike identification and analysis of the spike integrals. Experimentally, three of the $20 \mathrm{~s}$ chronoamperograms that were recorded at a carbon fibre microelectrode in a solution containing 12 pM silver nanoparticles and $20 \mathrm{mM} \mathrm{KCl}$ were analysed at five different frequencies: raw (ca. $2 \mathrm{k}$ $\mathrm{Hz}$ ), 500, 100, 50, and $25 \mathrm{~Hz}$. The resulting charge distribution at each frequency is presented as the cumulative frequency as a function of the measured charge. First, the total number of spike features detected in the experimental data is sensitive to the filtering frequency; this is depicted visually in the inlay of Figure 4. In the raw data a total of 254 individual events are detected but as the used filtering frequency is decreased, this number drops to 171 events at a filtering frequency of $25 \mathrm{~Hz}$. This decrease in the number of spikes detected is attributed to the merging of individual spikes into single features when observed in the filtered data set, akin to the example presented in Figure 2. Concomitantly, the merging of individual spikes together leads to an increase in the average charge passed per feature (see inlay of Figure 4). As such the use of filtering serves to group spikes that occur close in time together as single features. For the presented experimental data this change in 
the average charge passed is significant where for the raw data $0.211 \pm 0.012 \mathrm{pC}$ of charge is passed per electrochemical event and at a filter rate of $25 \mathrm{~Hz}$ this increases to $0.323 \pm 0.020 \mathrm{pC}$. The standard deviations for the raw data and $25 \mathrm{~Hz}$ filtered data are $0.195 \mathrm{pC}$ and $0.266 \mathrm{pC}$ respectively. Note the standard deviation does not solely represent the measurement error but predominantly reflects the large variability of the magnitude of the impact events as anticipated for a heterogeneous nanoparticle sample. Importantly, due to the relatively large numbers of individual events measured, the standard error of the mean is comparatively small relative to the mean. This highlights the significance of the differences between the charge distributions measured at the different filtering frequencies.

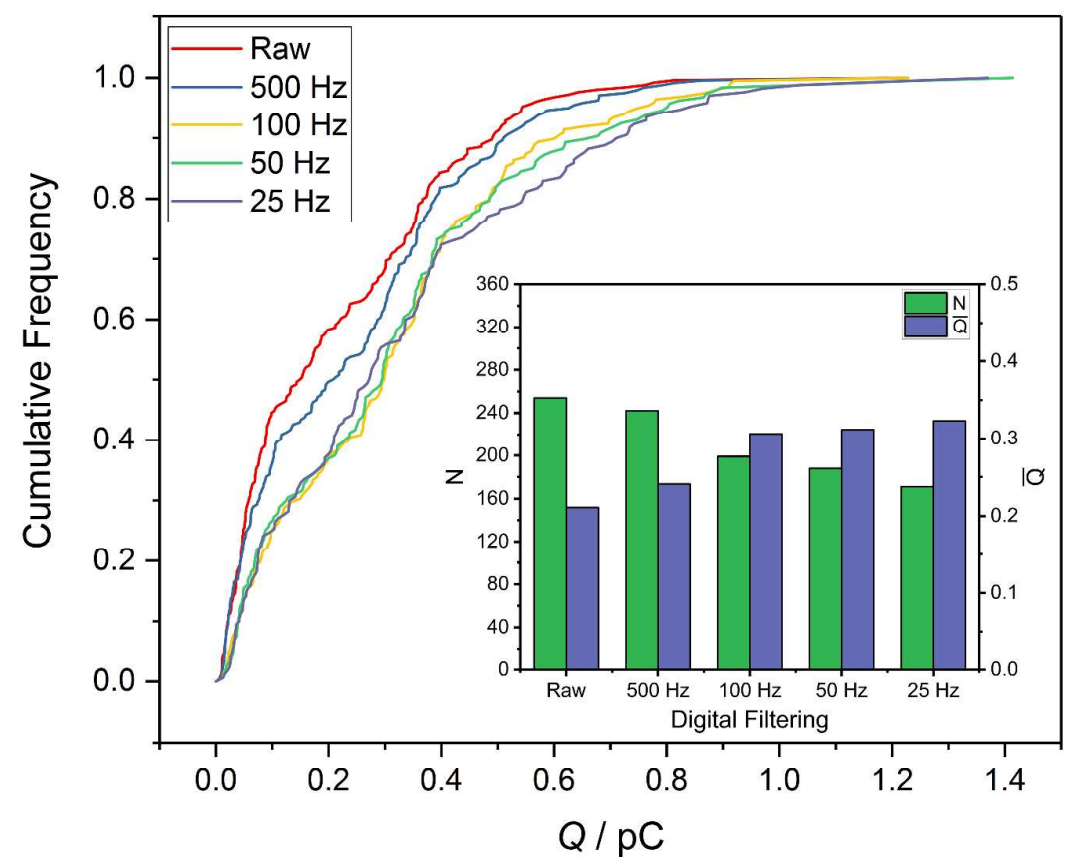

Figure 4. Cumulative frequency plots of the charge transferred during nano-impacts of $12 \mathrm{pM} \mathrm{AgNPs} \mathrm{in} 20 \mathrm{mM} \mathrm{KCl}$ at different filtering frequencies for 3 chronoamperograms. The red line depicts the distribution obtained from the raw data, whilst the blue, yellow, green and purple lines show the effect of filtering at 500,100, 50 and $25 \mathrm{~Hz}$ respectively. The inlay shows the effect of the filtering frequency on the number of individual events observed (green) and the value of the mean charge (purple)

The sensitivity of these results to the used low-pass filtering frequency raises a significant challenge. For data represented at high frequencies where individual electrochemical nano-impacts may exhibit multiple spikes, how can we determine which features correspond to the same interfacial event? Conversely, if the filtering frequency is too low do independent events become merged into being a single feature in the electrochemical data? Due to the arrival of the nanoparticles at the interface being a random event, it is impossible in the absence of more information to determine 
categorically if two spikes are associated with the same nano-impact or if they are independent. However, statistics may be employed to provide further insight. For a given particle concentration, known particle size and under the assumption the particles are consumed at the interface, the rate at which the nanoparticles are predicted to impact at a surface may be estimated. Under conditions of a steady-state flux to the electrochemical interface the rate of particle arrival is given by;

(1) $J(t \rightarrow \infty)=4 D C r_{d}$

In the above equation the particle diffusion coefficient can be readily estimated from the StokesEinstein equation as given by;

(2) $D=\frac{k_{B} T}{6 \pi \eta r_{N P}}$

Assuming that the nano-impact events are independent, the Poisson distribution enables us to determine the probability of observing two or more independent events in a given time period. Alternatively, we can calculate a period of time for which there is a $95 \%$ or $99 \%$ probability that maximally only one event is predicted to occur. For a $12 \mathrm{pM}$ solution of $50 \mathrm{~nm}$ diameter silver nanoparticles at room temperature and using an electrode of radius $16.5 \mu \mathrm{m}$, the predicted steadystate impact frequency is $4.7 \mathrm{~Hz}$. Consequently, on the basis of the Poisson distribution, there is a $99 \%$ probability that one or zero spike events will occur within a $31.5 \mathrm{~ms}$ window. For the 4-pole digital Bessel filter used in this work and with a low-pass filter frequency of $100 \mathrm{~Hz}$, the duration of the impulse response is ca. $15 \mathrm{~ms}$. Consequently, on the basis of the above argument, it is a reasonable inference that the use of a $100 \mathrm{~Hz}$ filtering frequency, for this present nanoparticle concentration and size, will not lead to a significant number of individual nano-impact events being merged together as a single feature. It should be noted that due to the random and stochastic nature of the impact events it is impossible, no matter the measurement frequency used, to exclude the possibility that two or more events may occur simultaneously and independently at the electrochemical interface. 


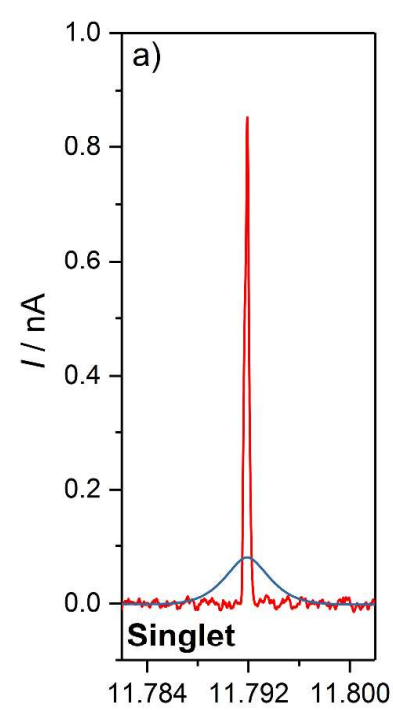

$t / \mathrm{s}$

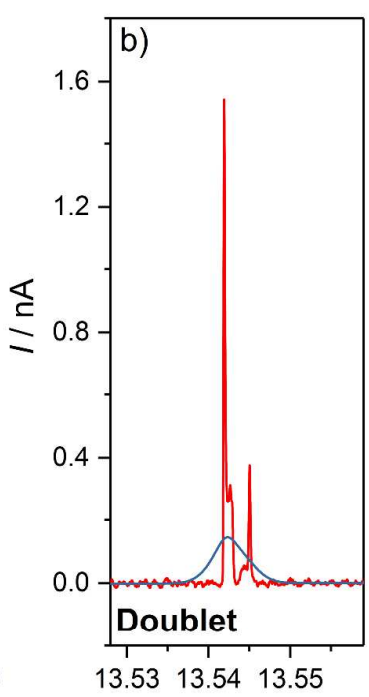

$t / s$

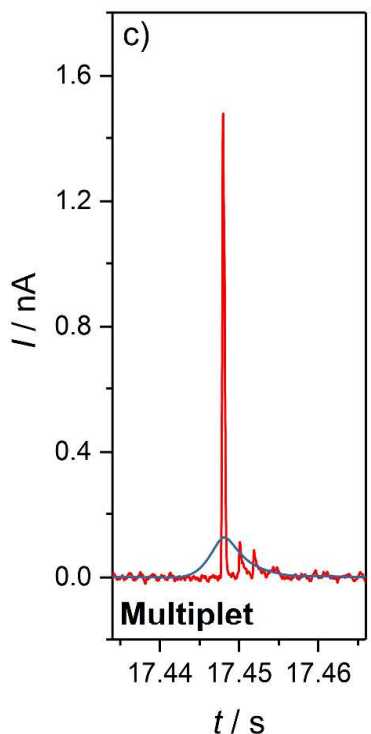

$t / \mathrm{s}$

Figure 5. Representative baseline-subtracted examples of 'singlet'(a), 'doublet' (b), and 'multiplet' (c) spikes for 12 pM AgNPs in $20 \mathrm{mM} \mathrm{KCl}$ at a carbon microdisc electrode (33 $\mu \mathrm{m}$ diameter). The raw data (red), is shown with the data filtered at $100 \mathrm{~Hz}$ (blue) overlaid. The singlet charge of $0.353 \mathrm{pC}$ measured at $100 \mathrm{~Hz}$ is comparable with the raw charge of $0.351 \mathrm{pC}$. For the doublet, the charge of $0.747 \mathrm{pC}$ measured at $100 \mathrm{~Hz}$ is also comparable with the raw charge of $0.752 \mathrm{pC}$, made up of two component charges of $0.603 \mathrm{pC}$ and $0.149 \mathrm{pC}$. Finally, the multiplet charge measured at $100 \mathrm{~Hz}$ of $0.705 \mathrm{pC}$ is slightly larger than the raw charge of $0.638 \mathrm{pC}$, made up of constituent charges of $0.522 \mathrm{pC}$, $0.062 \mathrm{pC}$ and $0.054 \mathrm{pC}$.

Having ascertained that the use of a $100 \mathrm{~Hz}$ filter is acceptable for the present work, we now seek to compare the same data set when viewed and analysed at its full $(2 \mathrm{kHz})$ and filtered $(100 \mathrm{~Hz})$ frequencies. Figure 5 represents 3 different individual nano-impact events; overlaid is the same data as represented in the raw data set and as reported after filtering to $100 \mathrm{~Hz}$. In Figure $5 \mathrm{a}$ ), both the raw data and the $100 \mathrm{~Hz}$-filtered data show a single spike. Moreover, the integral of these two peaks is near identical when measured at the two different frequencies $(100 \mathrm{~Hz}$ Charge: $0.353 \mathrm{pC}$, Raw Charge: $0.351 \mathrm{pC}$ ). Figure $5 \mathrm{~b}$ ) shows a single peak at $100 \mathrm{~Hz}$ but two separate spikes in the raw data. Note, as before, a single spike is defined by the current not crossing the baseline. Again the integral of the feature measured at $100 \mathrm{~Hz}(0.747 \mathrm{pC})$ is comparable to the sum of the charge passed during the two individual events as measured from the raw data (total charge $=0.752 \mathrm{pC}$ ). Finally, and in contrast for the nano-impact event depicted in Figure $5 \mathrm{c}$ ), at $100 \mathrm{~Hz}$ a single spike is observed (charge passed $0.705 \mathrm{pC}$ ) but in the raw data 3 individual spikes are observed. A fourth possible spike is also shown but the height of this process is below the required threshold for spike identification and hence is not included in the charge summation. Summation of the charge for the three individual events leads to a calculated total charge of $0.638 \mathrm{pC}$, i.e. approximately $10 \%$ less than found for the event when analysed at $100 \mathrm{~Hz}$. This issue of charge being partially 
underestimated for individual events when measuring data at a higher frequency and summing the charge passed during individual events is also reflected in the data analysis when performed on a larger population size. SI section 4 depicts comparative data where the charge distribution has been analysed from the detection of spike events in the raw data, then the raw data has subsequently been clustered together on the basis of a windowing method. Here, if further nano-events occur within 15 ms of the start of the first, the spikes are combined; this value is comparable to the duration of the impulse response of the filter. On average, measuring the data in this way at $2000 \mathrm{~Hz}$ results in the total mean spike charge being underestimated by approximately $5 \%$ compared to analysis of the data at $100 \mathrm{~Hz}$, excluding those spikes which 'vanish'. However, in this population of events, the charge associated with some spikes is in very significant error (ca. $70 \%$, see SI section 4 for more information). Moreover, the use of the of high frequency data leads to some features not being identified. As highlighted in the example shown in Figure 3, this occurs when the electrochemical process is slower, with a longer spike duration leading to a broad and relatively poorly defined peak in the chronoamperometry. As a preliminary conclusion, when the dynamics and kinetics of the nano event are of experimental interest, the use of higher frequency data is necessitated. Conversely, for situations in which the charge passed during the course of a nano-event is of interest, the analysis of data filtered to lower frequencies is more appropriate. Whilst the use of filtering to effectively cluster and merge spikes together as single events is challenging to justify on the basis of the statistics of the Poisson distribution (as compared to a windowing type method as exemplified in the SI section 4), the significant advantage of filtering the data set is the improvement in the signalto-noise ratio for slow electrochemical events. Crucially, the use of digital filtering enables both data sets to be made available from a single experiment, allowing more complete analysis.

At higher measurement frequencies, the chronoamperometric data reveals that individual nanoimpact events are regularly comprised of multiple spikes in current; as has been exemplified in the data presented in Figure 5. Comparison of the data at a given high and low frequency yields a route by which the multiplicity or the 'number of spikes per nano-impact event' can be systematically quantified. Here, the spike multiplicity gives a measure of the complexity of the current shape of the nano-impact event. In the following, the charge and duration of a nano-event is assessed from the data when it has been digitally filtered to $100 \mathrm{~Hz}$. Subsequently, the number of spike-like features per event can then be assessed by comparison of this data to that obtained at a higher frequency. For example, using this definition, the nano-impact events depicted in Figure 5 a), b) and c) are classified as a singlet, doublet and multiplet respectively, where a multiplet is defined as having 3 or more spike-like features per event. 
Figure 6 a) shows the distribution of the nano-impact events as categorised by the above multiplicity definitions. Of the 601 nano-impact events considered in total, 52\% are singlets when viewed in the raw data. Strikingly, of these nano-impact events, $26 \%$ of these features are not observable in the raw data (labelled 'vanished'); they are submerged by the noise of this system at the higher frequency. Figure $6 \mathrm{~b}$ ) presents the cumulative frequency for the four categories, showing the associated charge distributions. First, it can be seen that the features that are not detectable in the high frequency data are on average significantly smaller (considering mean total charge passed, $0.0435 \pm 0.0042 \mathrm{pC}$ ). This observation predominantly reflects the fact that the lower filter frequency has decreased the noise of the signal, enabling slow (and hence lower current magnitude) features to be resolved in the data set. However, the slow nature of these electrochemical features is of interest and may indicate that the processes leading to their detection differ markedly from other types of nano-impact events. Second, the charge distributions for the singlet, doublet and multiplet features are broadly comparable, but the average charge per event increases with the number of features present per event. Considering the data set in its entirety, the average charge passed for the whole population of events is found to be $0.223 \pm 0.009 \mathrm{pC}$. The following section serves to evidence to what extent the individual nanoparticle oxidation processes goes to completion.

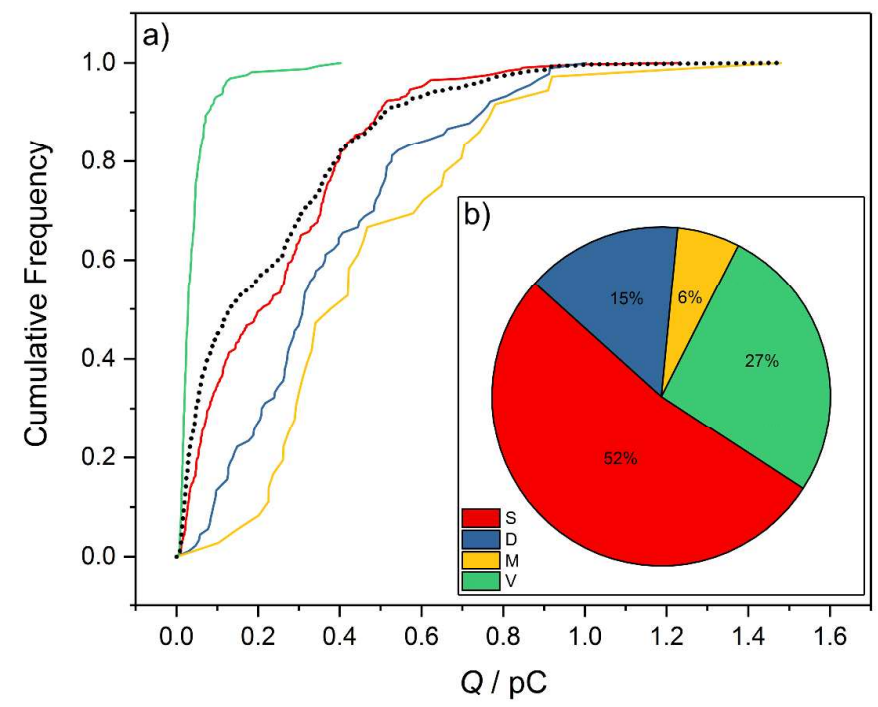

Figure 6. a) Cumulative frequency charge distributions of nano-impact events split by multiplicity by comparing raw data with that filtered at $100 \mathrm{~Hz}$. Singlets, doublets, multiplets and vanishing spikes are depicted by red, blue, yellow and green lines respectively, with the dotted black line showing the overall distribution. b) A pie chart showing the distribution of multiplicities of events when filtered at $100 \mathrm{~Hz}$.

After experimentally obtaining the nano-impact data, filtering the raw data to group processes occurring close together in time, removing the slowly changing background from the chronoamperometric data (baseline correction), identifying the individual nano-events and integrating the individual charges, it is vital to consider whether the observed individual nano- 
impact events are consistent with partial or total oxidation of the nanoparticles upon electrical contact with the electrode. To answer this question, one must consider both the average charge passed per nano-impact event, but also the prospect of information being contained in the experimental nano-impact frequency data.

First, as highlighted in the introduction, it is important to recognise that the electrochemical nanoimpact methodology is biased towards the detection of smaller nanoparticles due to their higher associated diffusion coefficient. If each nano-impact event is assumed to provide a measure of the impacting nanoparticles volume, we can also assume that this volume, $V$, gives a direct estimate of the diffusion coefficient of the particle (using a spherical approximation). Furthermore, if the probability of observing a nanoparticle is taken to be proportional to its diffusion coefficient (i.e. assuming a convergent steady-state mass-transport profile is operative), then the electrochemically measured charge distribution can be corrected by weighting each particle by the cube-root of the charge passed during the course of the individual nano-impact event $\left(\mathrm{Q}^{1 / 3}\right)$. This weighting derives from the Stokes-Einstein Equation (given in Eq. 2), where $D \propto \frac{1}{r}$ and, assuming a spherical nanoparticle, $V \propto r^{3}$, hence $D \propto \frac{1}{V^{1 / 3}}$. The influence of this diffusional correction is shown in Figure 7 such that the weighted data gives - under the approximations made above - a non-biased estimate of the total charge per nanoparticle in the solution phase. This is equivalent to the estimation of the solution phase nanoparticle volume distribution under the assumption of a known material density and with the application of Faraday's first law. The above discussion explicitly highlights an assumption made in using the electrochemical method as a route to providing a size estimate of the solution phase nanoparticle volume distribution. Comparison of this estimated volume distribution to that of TEM requires the construction of a volume distribution from 2D representations of the nanoparticle population. As outlined above, in the absence of further topographical or $3 \mathrm{D}$ information, significant assumptions regarding particle geometry are required to make an estimate of the nanoparticle volume distribution. TEM images of the nanoparticle sample used in this work demonstrate that this sample, as is commonly encountered in nanoparticle populations, exhibits significant heterogeneity both in terms of the particle dimension and morphology. From these images an estimate of the particle volume distribution and hence oxidative charge per particle can be made. In making this estimate a correction factor (f), as defined in previous work $^{30}$, is utilised and gives a guide to the possible error associated with the conversion of TEM images into a charge estimate per particle. The correction factors used are further discussed in the SI section S5. To re-emphasise this point, the circularity of a $2 \mathrm{D}$ projection of a particle in an 
image does not provide a good estimate of the particles sphericity, even highly symmetric shapes can have significantly lower volumes as compared to the circumscribed sphere.

Figure 7 depicts the TEM and electrochemical estimates of the equivalent charge distribution for the nanoparticle population. In accounting for the bias of the electrochemical technique towards the detection of smaller particles, the average charge per particle is determined to be $0.31 \mathrm{pC}$, with a standard deviation of $0.25 \mathrm{pC}$. Depending on the methodology used to estimate the charge per particle from the TEM data (see SI section S5), the expected charge per particle is in the range of $0.28 \pm 0.02$ to $0.32 \pm 0.02 \mathrm{pC}$. The mean charge per particle, as estimated by the two techniques, is found to be in excellent agreement. A minor discrepancy arises in the fact that the electrochemical data estimates a wider size distribution as evidenced by the increase in the reported standard deviation from $0.16 \mathrm{pC}$ (TEM) to $0.25 \mathrm{pC}$. Consequently, within the accuracy of the technique and the assumptions employed, the observed electrochemical data is consistent with the charge estimate made from the TEM data. This indicates that the nanoparticle oxidation goes to completion for this silver nanoparticle population in the presence of $20 \mathrm{mM} \mathrm{KCl}$. Previous work studying larger ca. 100 $\mathrm{nm}$ diameter particles has highlighted that in the larger size limit, the propensity for a particle to undergo complete or partial oxidation is likely dependent upon a variety of experimental factors, including the electrolyte identity and concentration. ${ }^{31}$ Having accounted fully for all of the variables and biases in the system and having analysed the data thoroughly, there is insufficient evidence to prove the nanoparticle population only undergoes partial oxidation at the electrochemical interface under these experimental conditions. 




Figure 7. Cumulative frequency charge distribution calculated from TEM sizing (assuming spherical nanoparticles) depicted as black dotted line with shaded area enclosed with a red line determined by the correction factor used. The purple dashed and solid lines show the unweighted and weighted charge distributions obtained from the chronoamperometry experiments respectively. Inlay depicts the mean cumulative number of impacts as a function of time for six chronoamperograms (red line) of 12 pM AgNPs in $20 \mathrm{mM} \mathrm{KCl,} \mathrm{with} \mathrm{the} \mathrm{associated} \mathrm{standard} \mathrm{error} \mathrm{bars.} \mathrm{The}$ green dashed line portrays the expected cumulative number of spikes assuming a steady-state flux to the electrode.

An alternate route to determine whether the nanoparticle oxidation goes to completion is to study the observed nanoparticle impact frequency. The frequency of the nanoimpact events can be measured and compared to that expected on the basis of a diffusional model. Were the nanoparticles to undergo multiple partial oxidations, one may anticipate the measured nano-event frequency to be markedly higher than that predicted on the basis of a diffusion-only model. However, such analysis is complicated by the fact that a number of factors, including particle adsorption onto a supporting surface, agglomeration or chemical kinetic processes can lead to the observed nanoparticle impact frequency being significantly less than would be initially predicted utilising a diffusion-only model of the nanoparticle mass-transport to the electrode surface..$^{30,32-33}$ The Figure 7 inlay depicts the mean cumulative number of spikes observed as a function of time for 6 separately measured chronoamperograms with the associated mean standard error. Also depicted is the theoretically predicted cumulative number of spikes as estimated from assuming a steady-state flux of material to the electrode surface. Although at short-times ( $<8$ seconds) the frequency of the nano-impacts is 
highly comparable to that predicted for a diffusion-only flux of material to the electrode surface, at longer times ( $>8$ seconds) the impact rate becomes significantly depleted. As has been previously reported, the observed nano-impact frequency of the oxidation of silver nanoparticles is sensitive to both the identity and concentration of the halide anion. Notably, the lower the halide concentration the lower the nano-impact frequency is observed to be (even in the presence of a constant ionic strength). Moreover, although the frequency of the impacts is sensitive to the halide concentration and identity, the magnitude of the average charge passed per event is found to be relatively invariant. This observation has been previously attributed to a nucleation growth type mechanism for the nanoparticle oxidation. ${ }^{30}$ In conclusion, in accounting for the complications outlined above, the impact frequency data presented here is not inconsistent with the interpretation that the nanoparticle oxidation goes to completion, given that a wide number of factors can and do influence the observed electrochemical response.

\section{Conclusions}

In accordance with the literature, the use of high measurement frequencies (ca. kilo hertz) reveals that individual nano-impact events tend to exhibit multiple maxima and in many cases are comprised of multiple spike features. This variation in the nano-impact event shape may in some cases reflect the underlying kinetics of the electrochemical reaction, or may reflect the nano-motion of the nanoparticle at the electrochemical interface. The value of the generic methodology employed in this work has been exemplified in the demonstration of the complete oxidation of silver nanoparticles of diameters as large as $50 \mathrm{~nm}$, and such data has been correlated with TEM images to indicate the non-spherical geometry of such particles. However, when the charge per nano-event is of interest, this work demonstrates that the use of higher measurement frequencies tends, even with the use of appropriately fast electronics and sampling, to result in the underestimation of the charge passed per nano-impact event. This occurs due to the fact that at higher measurement frequencies individual nano-events can be comprised of multiple spike features and the clustering of individual events together as single events is required to provide an estimate of the total charge passed per event. Moreover, higher signal bandwidths necessitate larger background noise and consequently slow and small processes can become 'lost' in the noise of the system. Consequently, in order to obtain more accurate information regarding the both the nano-impact event frequency and the charge passed per event, low-pass filtering the data to lower frequencies is advised prior to charge analysis. This filtering may be performed either prior to or after digitalisation of the signal. In order to exemplify the effect of the filtering system, this work has focused on digital filtering following data acquisition. The lowest suitable filtering frequency 
depends upon the expected nano-impact event frequency. Use of lower filtering frequencies will increase the probability of two independent events being counted as a single feature. However, for many experiments where frequencies of ca. $<5$ particle impact events are anticipated to occur per second, the use of a $100 \mathrm{~Hz}$ filtering frequency is acceptable and both reduces the electrochemical noise and facilitates quantitative analysis of the oxidative charge passed per event. Given the similarity of the duration of nanoparticle-electrode events in the same solvent, the results presented here have wider implications concerning the importance of considering the filtering frequency when pursuing quantitative charge information for a wide variety of nanoparticle systems. Namely, the use of a $100 \mathrm{~Hz}$ filtering frequency is widely suitable for charge analysis in this class of experiment.

\section{Experimental}

\section{Chemical Reagents}

Spherical citrate-AgNPs of ca. $50 \mathrm{~nm}$ diameter (Nanoxact, $0.02 \mathrm{mg} \mathrm{mL}^{-1}$ silver, $2 \mathrm{mM}$ sodium citrate) were purchased from Nanocomposix, USA. Potassium Chloride ( $\geq 99.0 \%$ ) was obtained from Sigma-Aldrich and was used as received without further purification. Solutions were prepared using deionized water (Millipore) with a resistivity of no less than $18.2 \mathrm{M} \Omega \mathrm{cm}$ at $25^{\circ} \mathrm{C}$.

\section{Nano-impacts}

Nano-impact experiments were carried out using a home-built in-house low noise potentiostat, as described previously. A low-noise current amplifier (LCA-4K-1G, FEMTO Messtechnik GmbH, Germany) was filtered using two cascade analog RC-filters at $2000 \mathrm{~Hz}$. The signal was then digitised at $100 \mathrm{KS} \mathrm{s}^{-1}$ via a USB data acquisition device (USB-6003, National Instruments, Texas, US). Finally, the signal was processed either in its raw form, or filtered digitally (4-pole Bessel) to frequencies of 25, 50,100, or $500 \mathrm{~Hz}$ using a script written in Python 3.5. Measurements were carried out at a carbon microdisc $(\mathrm{d}=33 \mu \mathrm{m}$, IJ Cambria Scientific Ltd, U.K.). We note that whilst this commercially supplied electrode nominally has a radius of $16.5 \mu \mathrm{m}$, on calibration it exhibited a radius of up to $22.6 \pm 1.3 \mu \mathrm{m}$. A leakless $\mathrm{Ag} / \mathrm{AgCl}$ (in $3.4 \mathrm{M} \mathrm{KCl}$, eDaq) electrode was used as a reference electrode, and a platinum mesh was used as a counter electrode. Chronoamperometry was performed on solutions containing $12 \mathrm{pM} \mathrm{AgNPs}$ and $20 \mathrm{mM} \mathrm{KCl}$ using the carbon microdisc working electrode. The potential was held at $0.8 \mathrm{~V}$ (vs. leakless $\mathrm{Ag} / \mathrm{AgCl}$ ) for $20 \mathrm{~s}$ in each case. Nano-impact spikes were identified and analysed using a script written in Python 3.5. 


\section{Acknowledgement}

The research is sponsored by the funding from the European Research Council under the European Union Seventh Framework Programme (FP/2007-2013)/ERC Grant Agreement No. [320403].

\section{References}

1. Micka, K. In Depolarisation of the dropping mercury electrode by suspensions of insoluble substances, 1960; p 1182.

2. Moller, G. In Grain Size Determination of Silver Halide Crystallites by Electrochemical Reduction, 1971;: p 29.

3. Robbs, P. H.; Rees, N. V., Nanoparticle electrochemistry. Physical Chemistry Chemical Physics 2016, 18 (36), 24812-24819.

4. Sokolov, S. V.; Eloul, S.; Kätelhön, E.; Batchelor-McAuley, C.; Compton, R. G., Electrodeparticle impacts: a users guide. Physical Chemistry Chemical Physics 2017, 19 (1), 28-43.

5. Batchelor-McAuley, C.; Ellison, J.; Tschulik, K.; Hurst, P. L.; Boldt, R.; Compton, R. G., In situ nanoparticle sizing with zeptomole sensitivity. Analyst 2015, 140 (15), 5048-5054.

6. Levin, B. D. A.; Padgett, E.; Chen, C.-C.; Scott, M. C.; Xu, R.; Theis, W.; Jiang, Y.; Yang, Y.; Ophus, C.; Zhang, H., Nanomaterial datasets to advance tomography in scanning transmission electron microscopy. Scientific data 2016, 3, 160041.

7. De Backer, A.; Jones, L.; Nellist, P. D.; Van Aert, S., Hybrid statistics-simulations based method for atom-counting from ADF STEM images. Ultramicroscopy 2017, 177, 69-77.

8. Anderson, P. M.; Guo, H.; Sunderland, P. B., Repeatability and reproducibility of TEM soot primary particle size measurements and comparison of automated methods. Journal of Aerosol Science 2017, 114, 317-326.

9. Sokolov, S. V.; Batchelor - McAuley, C.; Tschulik, K.; Fletcher, S.; Compton, R. G., Are Nanoparticles Spherical or Quasi - Spherical? Chemistry-A European Journal 2015, 21 (30), 10741-10746.

10. Kiejna, A.; Wojciechowski, K. F., Metal surface electron physics. Elsevier: 1996.

11. Rao, C. N. R.; Kulkarni, G. U.; Thomas, P. J.; Edwards, P. P., Metal nanoparticles and their assemblies. Chemical Society Reviews 2000, 29 (1), 27-35.

12. Plieth, W. J., Electrochemical properties of small clusters of metal atoms and their role in the surface enhanced Raman scattering. The Journal of Physical Chemistry 1982, 86 (16), 31663170 .

13. Villarreal, E.; Li, G. G.; Zhang, Q.; Fu, X.; Wang, H., Nanoscale Surface Curvature Effects on Ligand-Nanoparticle Interactions: A Plasmon-Enhanced Spectroscopic Study of Thiolated Ligand Adsorption, Desorption, and Exchange on Gold Nanoparticles. Nano Letters 2017, 17 (7), 4443-4452.

14. Asoro, M.; Damiano, J.; Ferreira, P. J., Size Effects on the Melting Temperature of Silver Nanoparticles: In-Situ TEM Observations. Microscopy and Microanalysis 2009, 15 (S2), 706-707.

15. Sokolov, S. V.; Tschulik, K.; Batchelor-McAuley, C.; Jurkschat, K.; Compton, R. G., Reversible or Not? Distinguishing Agglomeration and Aggregation at the Nanoscale. Anal Chem 2015, 87 (19), 10033-10039.

16. Saw, E. N.; Kratz, M.; Tschulik, K., Time-resolved impact electrochemistry for quantitative measurement of single-nanoparticle reaction kinetics. Nano Research 2017, 10 (11), 3680-3689. 
17. Ustarroz, J.; Kang, M.; Bullions, E.; Unwin, P. R., Impact and oxidation of single silver nanoparticles at electrode surfaces: one shot versus multiple events. Chemical science 2017, 8 (3), 1841-1853.

18. Oja, S. M.; Robinson, D. A.; Vitti, N. J.; Edwards, M. A.; Liu, Y.; White, H. S.; Zhang, B., Observation of multipeak collision behavior during the electro-oxidation of single Ag nanoparticles. Journal of the American Chemical Society 2016, 139 (2), 708-718.

19. Perera, R. T.; Rosenstein, J. K., Quasi-reference electrodes in confined electrochemical cells can result in in situ production of metallic nanoparticles. Scientific reports 2018, 8 (1), 1965.

20. Yakushenko, A.; Mayer, D.; Buitenhuis, J.; Offenhäusser, A.; Wolfrum, B., Electrochemical artifacts originating from nanoparticle contamination by $\mathrm{Ag} / \mathrm{AgCl}$ quasi-reference electrodes. $\mathrm{Lab}$ on a Chip 2014, 14 (3), 602-607.

21. Kätelhön, E.; Tanner, E. E. L.; Batchelor-McAuley, C.; Compton, R. G., Destructive nanoimpacts: What information can be extracted from spike shapes? Electrochimica Acta 2016, 199, 297-304.

22. Sakmann, B., Single-channel recording. Springer Science \& Business Media: 2013. 23. Yao, J.; Gillis, K. D., Quantification of noise sources for amperometric measurement of quantal exocytosis using microelectrodes. Analyst 2012, 137 (11), 2674-2681.

24. Zhou, X. S.; Mao, B. W.; Amatore, C.; Compton, R. G.; Marignier, J. L.; Mostafavi, M.; Nierengarten, J. F.; Maisonhaute, E., Transient electrochemistry: beyond simply temporal resolution. Chemical Communications 2016, 52 (2), 251-263.

25. Amatore, C.; Grün, F.; Maisonhaute, E., Electrochemistry within a Limited Number of Molecules: Delineating the Fringe Between Stochastic and Statistical Behavior. Angewandte Chemie International Edition 2003, 42 (40), 4944-4947.

26. Zhou, Y.-G.; Rees, N. V.; Compton, R. G., The Electrochemical Detection and Characterization of Silver Nanoparticles in Aqueous Solution. Angewandte Chemie International Edition 2011, 50 (18), 4219-4221.

27. Nyquist, H., Certain Topics in Telegraph Transmission Theory. Transactions of the American Institute of Electrical Engineers 1928, 47 (2), 617-644.

28. Shannon, C. E., A mathematical theory of communication. The Bell System Technical Journal 1948, 27 (3), 379-423.

29. Shannon, C. E., Communication in the Presence of Noise. Proceedings of the IRE 1949, 37 (1), 10-21.

30. Ngamchuea, K.; Clark, R. O. D.; Sokolov, S. V.; Young, N. P.; Batchelor - McAuley, C.; Compton, R. G., Single Oxidative Collision Events of Silver Nanoparticles: Understanding the Rate - Determining Chemistry. Chemistry-A European Journal 2017, 23 (63), 16085-16096. 31. Bartlett, T. R.; Sokolov, S. V.; Compton, R. G., Electrochemical Nanoparticle Sizing Via Nano - Impacts: How Large a Nanoparticle Can be Measured? ChemistryOpen 2015, 4 (5), 600605 .

32. Krause, K. J.; Brings, F.; Schnitker, J.; Kätelhön, E.; Rinklin, P.; Mayer, D.; Compton, R. G.; Lemay, S. G.; Offenhäusser, A.; Wolfrum, B., The Influence of Supporting Ions on the Electrochemical Detection of Individual Silver Nanoparticles: Understanding the Shape and Frequency of Current Transients in Nano-impacts. Chemistry - A European Journal 2017, 23 (19), 4638-4643.

33. Krause, K. J.; Yakushenko, A.; Wolfrum, B., Stochastic On-Chip Detection of Subpicomolar Concentrations of Silver Nanoparticles. Anal Chem 2015, 87 (14), 7321-7325. 